\title{
Boundary-layer transition over a rotating broad cone
}

\author{
K. Kato, ${ }^{1, *}$ P. H. Alfredsson,,${ }^{1,}$ and R. J. Lingwood ${ }^{1,2, \$}$ \\ ${ }^{1}$ Linné FLOW Centre, KTH Mechanics, SE-100 44 Stockholm, Sweden \\ ${ }^{2}$ Department of Mechanical and Aerospace Engineering, \\ Brunel University London, Uxbridge UB8 3PH, United Kingdom
}

(Received 26 March 2019; published 31 July 2019)

\begin{abstract}
The route to turbulence in the boundary layer on a rotating broad cone is investigated using hot-wire anemometry measuring the azimuthal velocity. The stationary fundamental mode is triggered by 24 deterministic small roughness elements distributed evenly at a specific distance from the cone apex. The stationary vortices, having a wave number of 24 , correspond to the fundamental mode and these are initially the dominant disturbanceenergy carrying structures. This mode is found to saturate and is followed by rapid growth of the nonstationary primary mode as well as the stationary and nonstationary first harmonics, leading to transition to turbulence. The amplitudes of these are plotted in a way to highlight the continued growth after saturation of the fundamental stationary mode.
\end{abstract}

DOI: 10.1103/PhysRevFluids.4.071902

The transition to turbulence in the rotating-disk boundary layer has been investigated since the 1940s [1,2]. In experiments one observes the development of stationary, with respect to the disk, corotating vortices in the boundary layer before transition to turbulence. Linear stability theory suggests that above a certain critical Reynolds number such vortices grow due to an inviscid convective cross-flow instability. The quite distinct transition Reynolds number found experimentally was suggested by Lingwood $[3,4]$ to be due to an absolute instability. Since then several researchers have studied this and other rotating flows in the BEK family (Bödewadt, Ekman, and Kármán) and a comprehensive review up to 2015 can be found in Ref. [5]. Slightly later the transition scenario on the disk was further investigated experimentally [6] and via direct numerical simulations [7], and it was conjectured that an absolute secondary instability on top of the primary vortices was likely to be the trigger for transition.

Here, we consider the flow driven by a cone rotating at a rate $\Omega^{*}$ in a still fluid ${ }^{*}$ denotes a dimensional quantity). The flow geometry is defined by the apex half-angle $\psi$ as shown in Fig. 1; $\psi=90^{\circ}$ gives the rotating-disk case. When the cone (or disk) rotates, the fluid at the surface is forced to move circumferentially. Fluid in the boundary layer is also transported radially resulting in an inflectional radial velocity profile. On a broad cone, where the cone-apex angle is large $\left(\psi \gtrsim 50^{\circ}\right)$, experiments show corotating vortices as for the disk case $\left(\psi=90^{\circ}\right)$ [8]. On the other hand, for sharp cones $\left(\psi \lesssim 40^{\circ}\right)$ centrifugal effects result in a Görtler type instability that dominates the flow [9]. Also for broad cones stability theory has shown that an absolute instability exists (see, for instance, Refs. [10,11]), similar to the one for the disk as suggested by Lingwood [4]. However, since the stationary corotating vortices always exist in a physical experiment the role or importance of this absolute instability with respect to transition is not clear [12].

\footnotetext{
*kentaro.kato@mech.kth.se

†phal@mech.kth.se

†rebecca.lingwood@brunel.ac.uk
} 


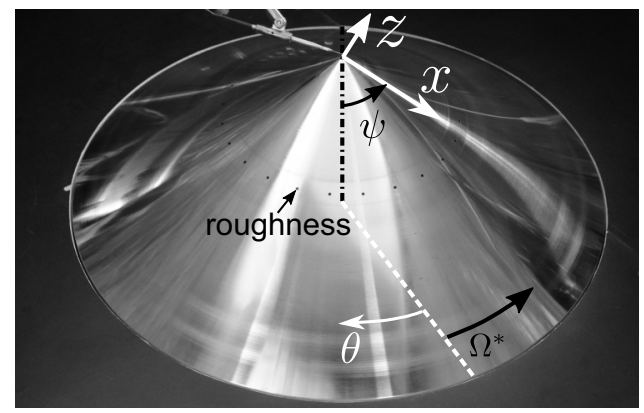

FIG. 1. The coordinate system $(x, \theta, z)$ on the rotating cone.

As shown in Fig. 1, an orthogonal coordinate system $(x, \theta, z)$ is defined on the cone surface with the origin located at the apex, where $x, \theta$, and $z$ are the coordinates along the generating line of the cone (herein, the radial direction), the circumferential direction, and the wall-normal direction. Lengths are normalized by a viscous length, $\delta_{v}^{*}=\sqrt{v^{*} /\left(\Omega^{*} \sin \psi\right)}$, where $v^{*}$ is the kinematic viscosity of the fluid and $x=x^{*} / \delta_{v}^{*}$ is the square root of the Reynolds number.

The experiments were conducted on a solid aluminum alloy cone, having a base diameter of $474 \mathrm{~mm}$ and an apex half-angle $\psi=60^{\circ}$. The cone surface has a smooth finish (surface roughness of $\sim 1 \mu \mathrm{m}$ ). It was mounted on an air bearing and rotated by a DC motor at $\Omega^{*}$ between 900 and $1800 \mathrm{rpm}$ around a vertical axis. The azimuthal velocity component was measured at a constant wall height $z=1.2$ using a single hot-wire probe with its sensing element parallel to the $x$ direction at fixed points in the laboratory frame. The length and diameter of the wire were approximately $0.5 \mathrm{~mm}$ and $2.5 \mu \mathrm{m}$, respectively. The signals from the anemometer and the tachometer attached to the spindle of the cone were simultaneously recorded for 1200 cone revolutions at a sampling rate of 720 data points per revolution. The velocity signal was postprocessed using a high-pass filter $\left(\omega^{*} / \Omega^{*}>3.5\right)$, where $\omega^{*}$ is the disturbance angular frequency (in the laboratory frame). Note that if the vortices are fixed with respect to the cone surface, then $\omega^{*} / \Omega^{*}$ gives the azimuthal wave number. In the following, the measured velocity was normalized by the local wall velocity $\Omega^{*} x^{*} \sin \psi$. From the azimuthal velocity fluctuation $v(t ; \theta, x)$, the stationary component $\tilde{v}(\theta ; x)$ was evaluated by phase averaging the fluctuation for every five revolutions. Further details of the setup as well as the mean flow can be found in Ref. [12].

The basic-flow characteristics of the cone flow are similar to that of the rotating disk (see Ref. [12]) except the most unstable wave number for the primary stationary disturbance, at the critical Reynolds numbers, is 22 for the disk and 19 for the $60^{\circ}$ cone. The corresponding critical $x$ values are 286 and 268, respectively. Here, we mounted 24 roughness elements uniformly in the azimuthal direction at a fixed radial location $x^{*}=115.7 \pm 0.5 \mathrm{~mm}$ to trigger the primary instability deterministically. We chose 24 roughness elements since experiments on the clean cone showed 20-26 vortices. As in Ref. [6] dry transfer lettering Letraset (Ref. 13045) was used, for the roughness, each was circular with a diameter of approximately $2 \mathrm{~mm}$. The deterministically introduced disturbance gives a fixed fundamental wave number on the entire cone [12] and its initial development compares well with linear stability analysis. In the case without deterministic disturbances, e.g., a clean cone or with randomly distributed roughness elements, the wave number may vary but the disturbance development is still well predicted by linear theory [13].

We present data from two different experiments: (i) for a given rotational speed, varying the roughness height $h^{*}$; and (ii) for a specific roughness height, varying the rotational speed. In case (i) the height is varied by layering elements, giving $h^{*}$ of approximately $4,8,13$, and $17 \mu \mathrm{m}$. In case (ii) $h^{*} \approx 8 \mu \mathrm{m}$ and $\Omega^{*}$ was $900,1200,1500$, or $1800 \mathrm{rpm}$. A case without roughness elements was also conducted, at the same four rotational speeds; the "clean" case.

Figure 2 shows the transition position, $x_{\mathrm{tr}}$, for all cases against the nondimensional roughness height $h=h^{*} / \delta_{v}^{*}$. Determining the transition position makes use of spectral information as 


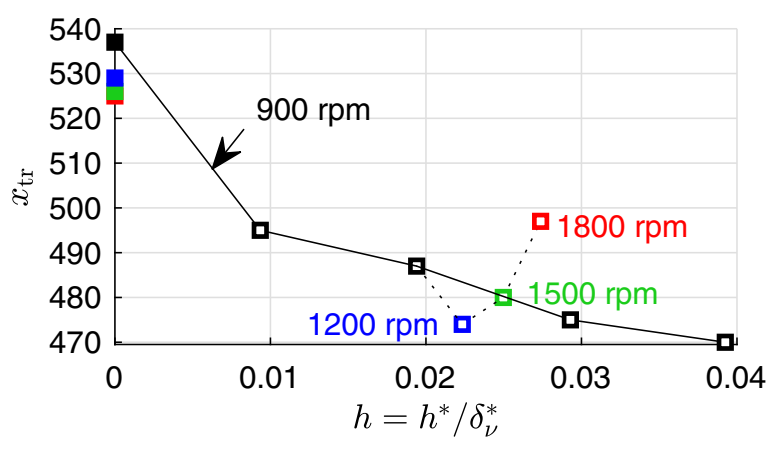

FIG. 2. Transition locations $x_{\text {tr }}$ against nondimensional roughness height $h=h^{*} / \delta_{v}^{*}$. Black, blue, green, and red markers correspond to 900, 1200, 1500, and $1800 \mathrm{rpm}$.

described below. The clean case corresponds to $h=0$ and $x_{\text {tr }}$ is seen to be rather insensitive to $\Omega^{*}$ although increasing $\Omega^{*}$ leads to slight decreases in $x_{\text {tr }}$. As expected, with increasing $h^{*}$ [case (i)], at a given rotational speed (here $900 \mathrm{rpm}$ ), the location of transition moves upstream (decreasing $x_{\mathrm{tr}}$ ), whereas the behavior with an increasing rotational speed and fixed roughness position and height [case(ii)], is nonmonotonic; this will be explained below.

The development of the disturbances will first be discussed using the power-spectrum development with $x$ as shown in Fig. 3. Here the power-spectrum density $\log (E)$ of the azimuthal velocity component for the cone rotating at $900 \mathrm{rpm}$ with (a) two layers and (b) four layers of Letraset and also in (c) the case with $1800 \mathrm{rpm}$ with two layers are presented. The ordinate shows normalized frequency $\omega^{*} / \Omega^{*}$. The solid line shows the neutral curve based on local stability analysis. The dashed lines indicate the location of the roughness elements. There is no unique way to determine the transition position. Here we use the spectral information to calculate the mean spatial (in $x$ ) growth rate of all wave numbers. The position where the mean growth rate reaches its maximum is taken as the transition location, $x_{\mathrm{tr}}$.

The roughness elements initially introduce disturbances at multiple harmonics $\left(\omega^{*} / \Omega^{*}=\right.$ $24,48, \ldots)$. In case (i), comparing Figs. 3(a) and 3(b), most of the disturbances disappear below $x \approx 300$ except the fundamental $\left(\omega^{*} / \Omega^{*}=24\right)$. Although the fundamental disturbance also decays, it begins to grow when entering the unstable region (beyond the neutral curve, i.e., $x>286$ ). Further downstream (i.e., larger $x$ ) higher harmonics appear and at a distinct $x$ the spectrum fills up indicating that transition has occurred. The comparison between Fig. 3(a) and 3(b) shows that increasing $h^{*}$ makes the harmonics appear at smaller $x$ locations and the transition shifts upstream.

Another way to study the transition scenario in more detail is to plot the development of the fundamental $\left(\omega^{*} / \Omega^{*}=24\right.$, dash-dotted lines $)$ and first harmonic $\left(\omega^{*} / \Omega^{*}=48\right.$, solid lines $)$ as in Fig. 4(a). As $h^{*}$ increases, the initial transient coupled to the disturbance increases. After a short transient for $x \lesssim 295$, the fundamental grows and the spatial growth rate shows good agreement with local linear stability analysis (LLSA) up to $x \approx 380$ in Fig. 4(b). In the linear region, the roughness height does not affect the growth rate but slightly affects the $x$ location where the growth rate begins to deviate from LLSA. The deviation occurs when $\tilde{v}_{\text {rms }}$ of the fundamental reaches a certain magnitude $\tilde{v}_{\text {rms }, 24} \approx 10^{-2}$ (or the first harmonic reaches $\tilde{v}_{\text {rms }, 48} \approx 10^{-4}$ ), similar to what Appelquist et al. reported (Figs. 8 and 9 in Ref. [7]) for the rotating disk. Around the deviation point, the first harmonic (solid line) has its maximum growth rate which is nearly double that of the fundamental, indicating a quadratic nonlinear process [14]. After nonlinear saturation, both the fundamental and the first harmonic reach their maxima in the range $484 \lesssim x \lesssim 512$, nearly corresponding to the transition locations marked by the arrows on the abscissa. Increasing the roughness height shifts the whole process upstream. 


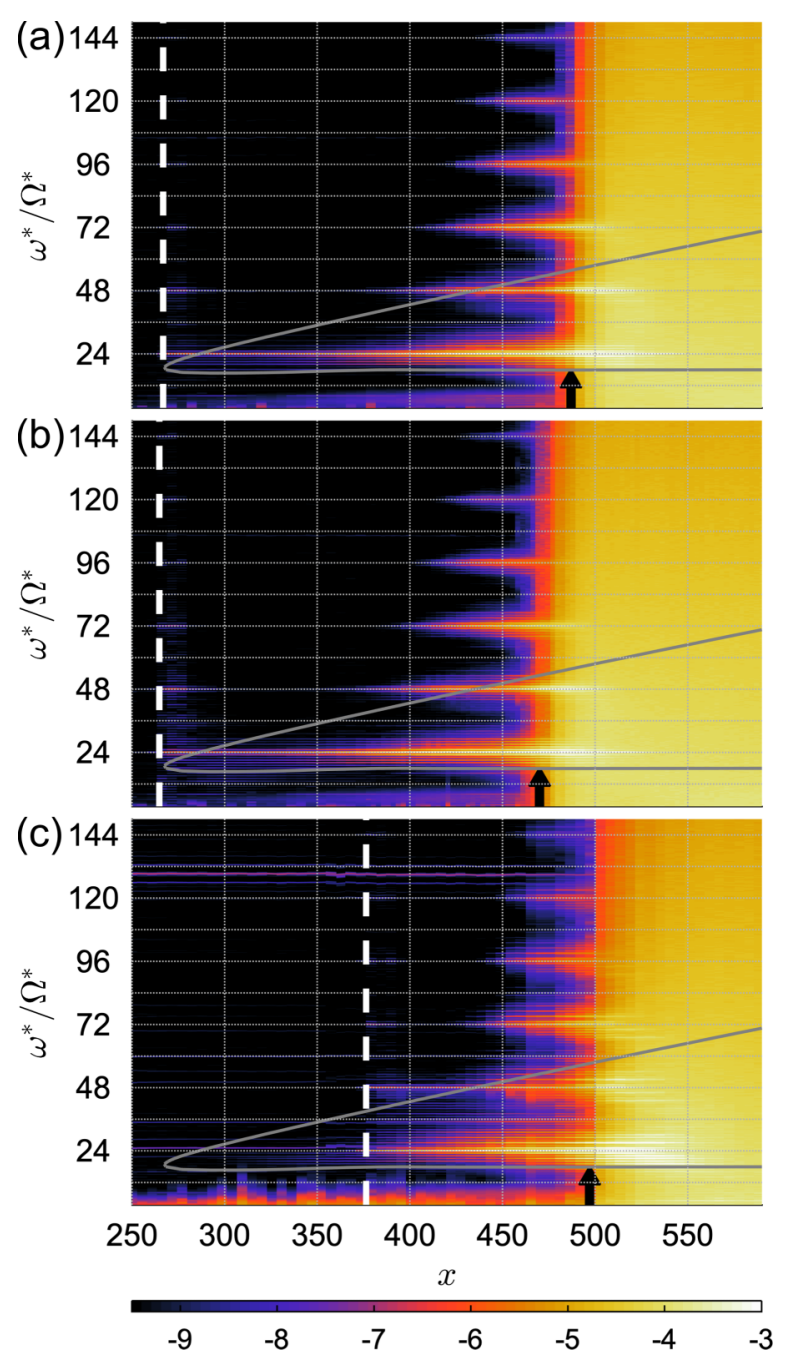

FIG. 3. Power-spectrum density $\log (E)$ for the cone with 24 roughness elements: (a) $h^{*}=8 \mu \mathrm{m}, 900 \mathrm{rpm}$; (b) $h^{*}=17 \mu \mathrm{m}, 900 \mathrm{rpm}$; and (c) $h^{*}=8 \mu \mathrm{m}, 1800 \mathrm{rpm}$. The solid line shows the neutral curve for stationary disturbances from linear stability theory. The dashed lines at (a) and (b) $x=267$, and (c) $x=377$ locate the roughness elements. The black arrow on the abscissa indicates the transition location for the respective case, as in Fig. 2.

We now focus on the power-spectrum density for case (ii) in Fig. 3 with a fixed roughness height ( $h^{*}=8 \mu \mathrm{m}$ ), but different $\Omega^{*}$ : (a) $900 \mathrm{rpm}$ and (c) $1800 \mathrm{rpm}$. When increasing the rotational speed the characteristic length scale $\delta_{v}^{*}$ decreases, which brings two effects: (i) increasing the amplitude of the initial disturbance $h^{*} / \delta_{v}^{*}$; and (ii) shifting the location of the initial disturbance downstream (with respect to the normalized $x$ location). In Fig. 3(c), the roughness is within the unstable region and the fundamental begins to grow directly; however, since the roughness element is at a larger $x$, the disturbance amplitude does not catch up with that introduced at smaller $x$ despite the nondimensional height of the roughness being larger. This leads somewhat counterintuitively to transition occurring around $x=497$ compared to $x=487$ at the lower rotational speed.

To get a better picture of the development of the amplitude of the fundamental and first harmonic of the stationary mode, we plot these as in Fig. 4. Figure 5 shows the development for the cases with different rotational speeds with fixed roughness height $\left(h^{*}=8 \mu \mathrm{m}\right)$. Here, it is clearly seen that for 

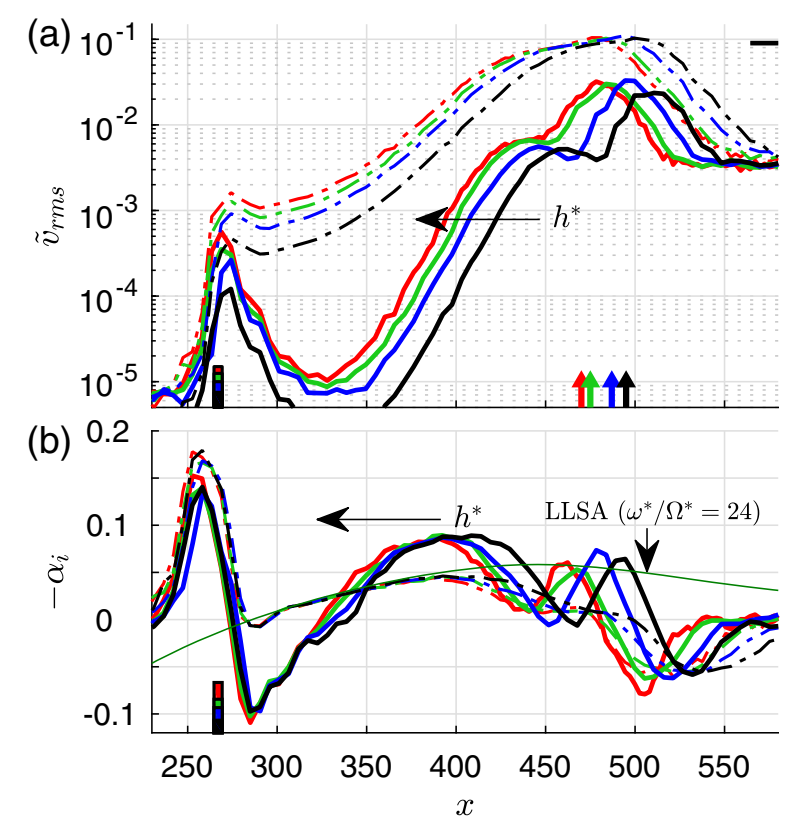

FIG. 4. (a) rms and (b) spatial growth rate $\left(-\alpha_{i}\right)$ of the azimuthal velocity fluctuation with different roughness heights (900 rpm): $h^{*}=4 \mu \mathrm{m}$ (black), $8 \mu \mathrm{m}$ (blue), $13 \mu \mathrm{m}$ (green), and $17 \mu \mathrm{m}$ (red). The rectangle at $x=267$ indicates the roughness elements. The dash-dotted and solid lines show the stationary components of the fundamental and the first harmonic $\left(\omega^{*} / \Omega^{*}=24\right.$ and 48 ), respectively. In (a), the solid marker (upper right) indicates the total rms level for the turbulent flow. The arrows on the abscissa in (a) show the transition locations as in Fig. 2; in (b), the thin line shows the growth rate based on LLSA.

$900 \mathrm{rpm}$ the fundamental decreases after the initial transient before it starts to amplify according to LLSA. However, for $1200 \mathrm{rpm}$ (where the roughness element is within the unstable region), the fundamental does not decay but amplifies directly after the initial transient from a larger amplitude than that of the $900 \mathrm{rpm}$ case at the same $x$ and, therefore, its amplitude leads the $900 \mathrm{rpm}$ case. For the two other cases (1500 and $1800 \mathrm{rpm}$ ), the initial transient becomes larger and they also amplify directly. In the case of $1500 \mathrm{rpm}$, by chance, the fundamental initially almost perfectly overlaps with the $900 \mathrm{rpm}$ case. For $1800 \mathrm{rpm}$, the initial transient is slightly higher but the development is shifted downstream compared to the others.

Just after the transient of each first harmonic, the initial decay rates follow LLSA $\left(\omega^{*} / \Omega^{*}=\right.$ 48) for all cases shown in Fig. 5(b). Then, the growth rates follow a similar pattern as for the fundamentals and their maximum growth rates are typically twice those of the fundamentals, except for $1800 \mathrm{rpm}$, which is slightly smaller. The transition positions shown by the arrows at the bottom of the figure seem to be coupled to the largest maxima of the harmonics.

From what has been shown above, it is clear that the stationary vortices have a role in the transition scenario and initially they are the dominant disturbance-energy carrier. In order to investigate the nonlinear interaction and transition process it is possible to plot harmonics as functions of this fundamental stationary mode (instead of plotting them against $x$ ). Some examples are shown in Figs. 6(a)-6(c) where the fundamental nonstationary mode as well as the first harmonic of the stationary and nonstationary modes are shown. The nonstationary component $v^{\prime}$ was obtained by subtracting the stationary component from the total fluctuation signal. Here, we show data for four different $\Omega^{*}$ and, after the initial transient, the disturbance amplitudes collapse for all three modes, albeit the highest rotational rate (red) has a slightly smaller slope [see also Fig. 5(b)]. The growth rate of the first harmonic of the stationary disturbance is double that of the fundamental 

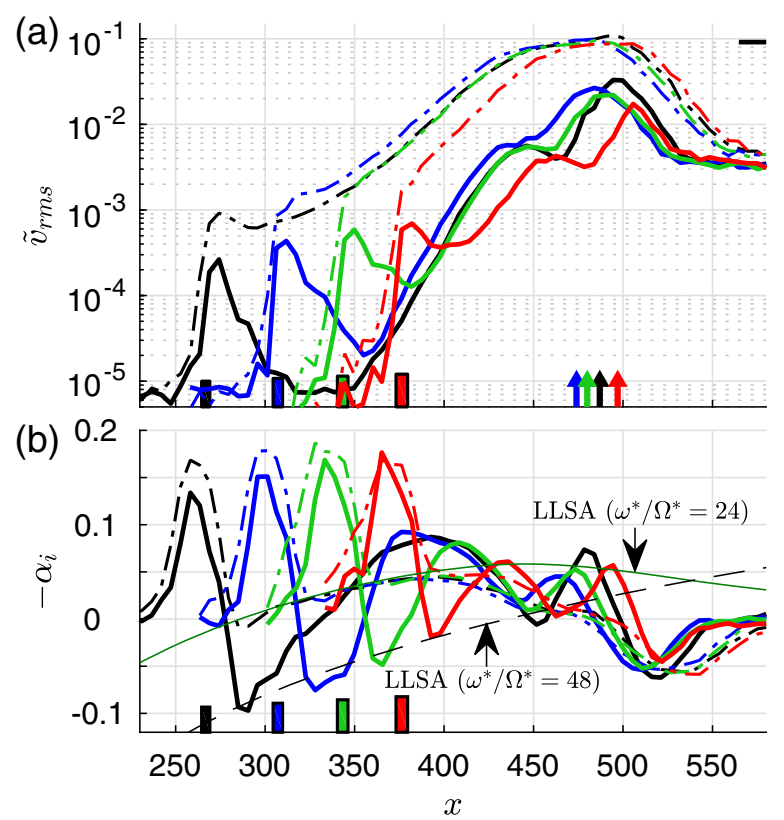

FIG. 5. (a) rms and (b) spatial growth rate $\left(-\alpha_{i}\right)$ of the azimuthal velocity fluctuation for different rotational speed cases $\left(h^{*}=8 \mu \mathrm{m}\right): 900 \mathrm{rpm}$ (black), $1200 \mathrm{rpm}$ (blue), $1500 \mathrm{rpm}$ (green), and $1800 \mathrm{rpm}$ (red). The rectangles at the bottom indicate the roughness elements. The dash-dotted and solid lines show the stationary components of the fundamental and the first harmonic $\omega^{*} / \Omega^{*}=24$ and 48, respectively. In (a), the solid marker (upper right) indicates the total $\mathrm{rms}$ level for the turbulent flow. The arrows on the abscissa show the transition location as in Fig. 2; in (b), the thin solid and dashed lines show the growth rate for the fundamental and first harmonic based on LLSA.

as indicated by the slope of 2 in the figure. A similar behavior can be seen for the nonstationary components.

Figures 6(d)-6(f) show the ratio of the amplitude of each mode to that of the fundamental stationary mode $\widetilde{v}_{\text {rms }, 24}$. As can be seen initially the nonstationary fundamental disturbance grows at the same rate as the stationary disturbance, i.e., the ratio of the nonstationary to the stationary fundamental disturbance remains a constant, nearly 10\% shown in Fig. 6(d). However, the first harmonic of the stationary disturbance as well as the nonstationary disturbances (both the fundamental and first harmonic) continue to grow when the fundamental mode has saturated $\left(\widetilde{v}_{\mathrm{rms}, 24} \approx 8 \times 10^{-2}\right)$. Eventually the nonstationary disturbances take over and dominate the disturbance energy; the amplitude ratio exceeds unity in Figs. 6(d) and 6(f); however, at that point transition has already occurred and disturbance energy has spread over the whole spectrum.

This behavior can, of course, also be observed in Figs. 4(a) and 5(a); however, by plotting the harmonics against the amplitude of the fundamental disturbance, this development becomes clearly illuminated.

The results presented here seem to indicate that as the stationary mode saturates, a mean-flow modification with a three-dimensional base flow establishes. At this stage, a clear change in energy growth is seen, from growth of the primary stationary vortices to a growing unsteady primary mode as well as stationary and nonstationary harmonics. These become the dominant growing energy carriers during this stage of transition to turbulence. Whether this is the same as the absolute secondary instability discussed in Ref. [7] or a different mechanism needs further investigation. 

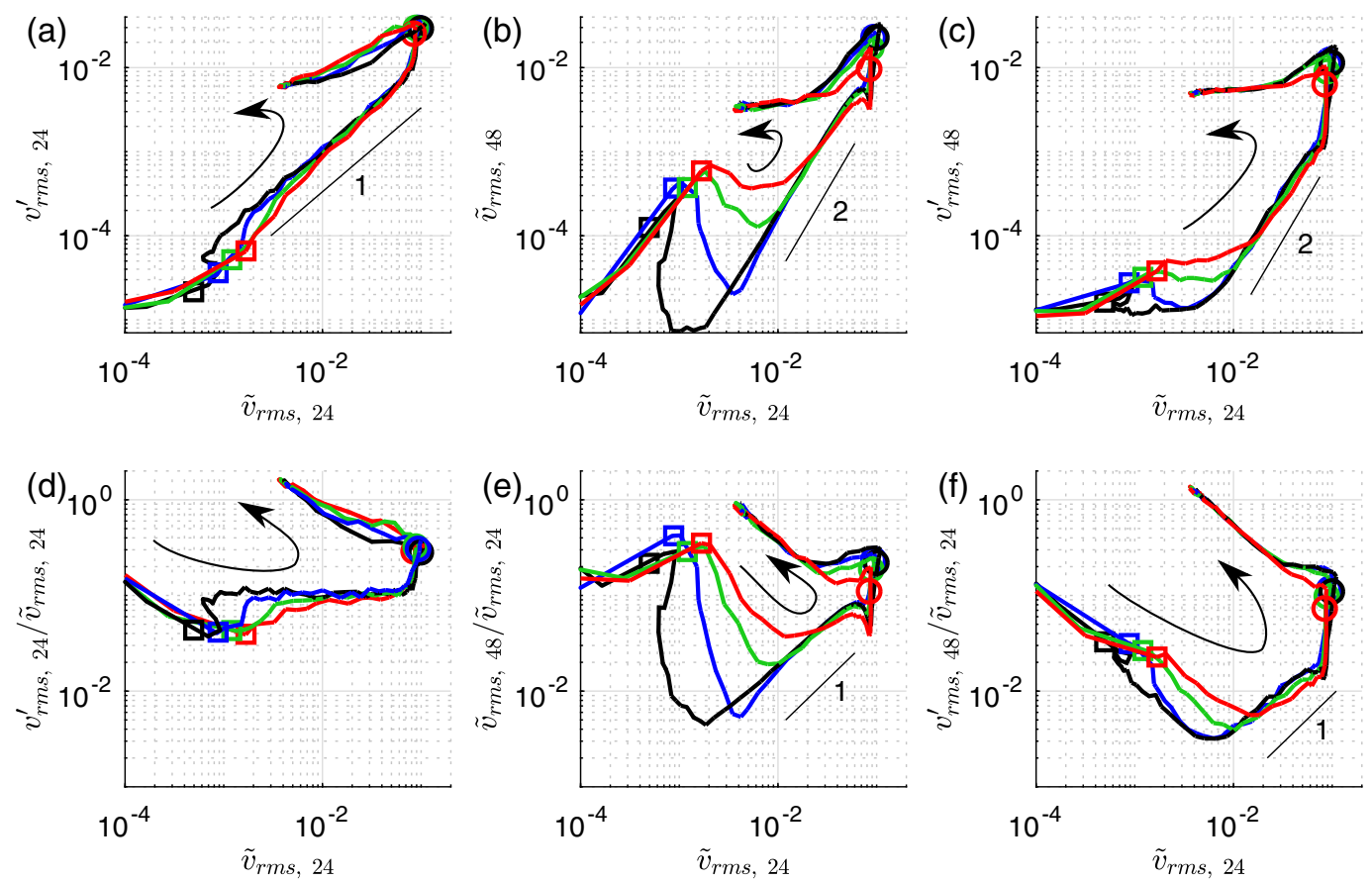

FIG. 6. Amplitude of different disturbance components as a function of the amplitude of the fundamental disturbance: (a) $v_{\text {rms } 24}^{\prime}$, (b) $\widetilde{v}_{\text {rms,48 }}$, (c) $v_{\text {rms } 48}^{\prime}$ and its ratio to the amplitude of the fundamental disturbance: (a) $v_{\text {rms }, 24}^{\prime} / \widetilde{v}_{\text {rms } 24}$, (b) $\widetilde{v}_{\text {rms }, 48} / \widetilde{v}_{\text {rms }, 24}$, (c) $v_{\text {rms }, 48}^{\prime} / \widetilde{v}_{\text {rms }, 24}$ for $\Omega^{*}: 900 \mathrm{rpm}$ (black), $1200 \mathrm{rpm}$ (blue), $1500 \mathrm{rpm}$ (green), and $1800 \mathrm{rpm}$ (red). The squares indicate the location of roughness elements and the circles the transition position. The anticlockwise arrows show the direction of increasing $x$.

We thank Dr. Antonio Segalini for providing the local linear stability analysis results. This work was supported mainly by the Swedish Research Council through the ASTRID project (VR Contract No. 2013-5786), supporting K.K.

[1] N. H. Smith, Exploratory investigation of laminar-boundary-layer oscillations on a rotating disk, NACA Tech. Note, No. TN 1227, 1947.

[2] N. Gregory, J. T. Stuart, and W. S. Walker, On the stability of three-dimensional boundary layers with application to the flow due to a rotating disk, Philos. Trans. R. Soc. London A 248, 155 (1955).

[3] R. J. Lingwood, Absolute instability of the boundary layer on a rotating disk, J. Fluid Mech. 299, 17 (1995).

[4] R. J. Lingwood, An experimental study of absolute instability of the rotating-disk boundary-layer flow, J. Fluid Mech. 314, 373 (1996).

[5] R. J. Lingwood and P. H. Alfredsson, Instabilities of the von Kármán boundary layer, Appl. Mech. Rev. 67, 030803 (2015).

[6] S. Imayama, P. H. Alfredsson, and R. J. Lingwood, Experimental study of rotating-disk boundary-layer flow with surface roughness, J. Fluid Mech. 786, 5 (2016).

[7] E. Appelquist, P. Schlatter, P. H. Alfredsson, and R. J. Lingwood, Transition to turbulence in the rotatingdisk boundary-layer flow with stationary vortices, J. Fluid Mech. 836, 43 (2018).

[8] R. Kobayashi, Review: Laminar-to-turbulent transition of three-dimensional boundary layers on rotating bodies, ASME J. Fluids Eng. 116, 200 (1994). 
[9] Z. Hussain, S. J. Garrett, and S. O. Stephen, The centrifugal intability of the boundary-layer flow over slender rotating cones, J. Fluid Mech. 755, 274 (2014).

[10] S. J. Garrett and N. Peake, The absolute instability of the boundary layer on a rotating cone, Eur. J. Mech. B Fluids 26, 344 (2007).

[11] C. Thomas and C. Davies, Global linear instability of rotating-cone boundary layers in a quiescent medium, Phys. Rev. Fluids 4, 043902 (2019).

[12] K. Kato, T. Kawata, P. H. Alfredsson, and R. J. Lingwood, Investigation of the structures in the unstable rotating-cone boundary layer, Phys. Rev. Fluids 4, 053903 (2019).

[13] E. Appelquist, S. Imayama, P. H. Alfredsson, P. Schlatter, and R. J. Lingwood, Linear disturbances in the rotating-disk flow: A comparison between results from simulations, experiments and theory, Eur. J. Mech. B Fluids 55, 170 (2016).

[14] M. Matsubara and P. H. Alfredsson, Secondary instability in rotating channel flow, J. Fluid Mech. 368, 27 (1998). 